\title{
ENC-MAC: Energy-efficient Non-overlapping Channel MAC for Cognitive Radio enabled Sensor Networks
}

\author{
Bosung Kim ${ }^{1}$, Kwangsoo Kim ${ }^{1}$, and Byeong-hee Roh $^{1}$ \\ ${ }^{1}$ Department of Computer Engineering, Ajou University \\ Suwon, Gyeonggi-do, 443-749, Republic of Korea \\ [e-mail: \{kbs8354, zubilan, bhroh\}@ajou.ac.kr] \\ *Corresponding author: Byeong-hee Roh
}

Received June 16, 2015; revised August 16, 2015; accepted September 16, 2015; published November 30, 2015

\begin{abstract}
The concept of Internet of Things (IoT) has shed new light on WSN technologies. MAC protocol issues improving the network performance are important in WSNs because of the increase in demand for various applications to secure spectrum resources. Cognitive radio (CR) technology is regarded as a solution to the problems in this future wireless network. In recent years, energy efficiency has become an issue in CR networks. However, few relevant studies have been conducted. In this paper, an energy-efficient non-overlapping channel MAC (ENC-MAC) for CR-enabled sensor networks (CRSNs) is proposed. Applying the dedicated control channel approach, ENC-MAC allows the SUs to utilize channels in a non-overlapping manner, and thus spectrum efficiency is improved. Moreover, the cooperative spectrum sensing that allows an SU to use only two minislots in the sensing phase is addressed to enhance energy efficiency. In addition, an analytical model for evaluating the performance, such as saturation throughput, average packet delay, and network lifetime, is developed. It is shown in our results that ENC-MAC remarkably outperforms existing MAC protocols.
\end{abstract}

Keywords: cognitive radio, energy efficiency, Internet of things, medium access control, spectrum efficiency, wireless sensor network

This work was supported by the National Research Foundation of Korea (NRF) grant funded by the Korea Government (MSIP) (no. NRF-2015R1A2A2A01005577). 


\section{Introduction}

Internet of Things (IoT) is an All Things on Networks (AToN) that are connected to all the information and knowledge of the world [1]. This concept is accomplished by utilizing mobile communications networks to deliver information. Among these networks, wireless sensor networks (WSNs) are currently used in domain-specific industrial applications such as smart power grids, intelligent transportation, structural health monitoring, environmental protection, smart agriculture, and disaster management [2][3].

In order to efficiently utilize spectrum resources, various kinds of multichannel MAC protocols for WSNs [4] have been proposed and are described in Section 2.1. They mostly considered energy efficiency because wireless sensor nodes are usually constrained in power supply [5][6]; however, there is little interest in the coexistence of multiple networks in the same license-free spectrum. According to Cisco, by 2020 there will be more than 50 billion devices connected to the Internet, which is seven times the world's population [7]. Thus, the spectrum shortage problem is also a critical issue for WSNs in which the devices are operated because these kinds of networks work in the license-free band, which is expected to suffer from heavy interference caused by other networks sharing the same spectrum band [8].

Cognitive radio (CR) technology is regarded as a very attractive solution to the spectrum inefficiency problem in these WSNs [9]. The CR-enabled sensor networks (CRSNs) correspond with distributed cognitive radio networks (DCRNs), where the unlicensed users, i.e., secondary users (SUs), can communicate through ad hoc connections [9]. The DCRN without central network units is one of the major network architectures applying the CR technology. Hence, the design of the MAC protocol for DCRNs is crucial, yet challenging, to increase the spectrum efficiency in wireless networks, given the dynamic nature of spectrum usage [10]. The influence of upper layer protocols is also an important design consideration for MAC protocols [11].

The MAC protocols for DCRNs introduced so far can be categorized into random access (contention-based), time-slotted (contention-free), and hybrid protocols [9][10]. Among them, the hybrid protocols try to achieve tradeoff between the random access and time-slotted protocols and thus are considered to be the most efficient spectrum access protocols.

However, most hybrid protocols for DCRNs [15]-[17] neglect energy efficiency, except for a few [18][19]. The hybrid protocols usually consist of four phases: sensing phase, reporting phase, contention phase, and data transmission phase. Depending on the spectrum sensing schemes, the reporting phase may not be considered in the design of hybrid protocols for DCRNs [16]. A number of hybrid protocols with these four phases have been proposed; however, there remains technical and structural room for improvement, which is described in Section 2.2.

In this paper, an energy-efficient non-overlapping channel MAC (ENC-MAC) for CRSNs is proposed. In order to process the control signaling and data transmission at the same time, the dedicated common control channel (CCC) approach is applied [23][24]. It is assumed that the SUs are equipped with two half-duplex transceivers to efficiently perform the four aforementioned phases. The sensing phase is designed such that each SU senses the states of two channels: a channel for report (CFR) and a channel for use (CFU). The channels are assigned by a master SU and by contention with other SUs, respectively. In the reporting phase, cooperative spectrum sensing is proposed for each SU to report only the assigned CFRs in its 
Received June 16, 2015; revised August 16, 2015; accepted September 16, 2015; published November 30, 2015corresponding minislot. In the contention phase, an SU contends with other SUs for a CFU among the available channels identified in the reporting phase, which is based on the IEEE 802.11 distributed coordination function (DCF) [25]. Thus, the SUs can use the channel in a non-overlapping manner. Further, an analytical model to evaluate the performance of the proposed scheme is developed in terms of saturation throughput, average packet delay, and network lifetime.

In Section 2, we review the MAC protocols in WSNs and CRNs. Sections 3 and 4 address the system model and overall operation process of the proposed scheme, respectively. Given the proposed scheme, Sections 5 and 6 present an analytical model and experimental evaluation for the proposed scheme, respectively. Lastly, we conclude this paper in Section 7.

\section{Related Work}

\subsection{Multichannel MAC Protocols in WSNs}

There has been a tremendous amount of research on the design and implementation of MAC protocols in WSNs. According to [4], MAC protocols for WSNs are classified into four categories: asynchronous, synchronous, frame-slotted, and multichannel. Recently, multiple channel MAC protocols have become a hot topic.

One challenge for future research is the design of dynamic allocation algorithms that adapt to the dynamic traffic of sensor networks. The interference analysis of prior work was based on network topology, and thus its solutions may not be the best fit for current interference conditions. It is desirable to allocate network resources flexibly according to the traffic, but it is also challenging to design such a dynamic channel allocation algorithm with low overhead.

Another challenge in multichannel MAC protocols is cross-channel communication. For cost considerations, a sensor node is usually equipped with a half-duplex radio transceiver that is unable to listen on multiple channels simultaneously. This implies that a node has to switch back and forth between two channels to check whether it has data to receive and whether the target receiver's channel is clear. These operations increase the overhead for packet transmission, and the frequent channel switching causes a node to consume more energy than other MAC protocols.

Reviewing the developed MAC protocols in WSNs so far, designers were mostly concerned with energy efficiency because sensor nodes are usually limited in power supply However, since the introduction of the IoT paradigm, wireless devices and applications have rapidly increased, and thus spectrum efficiency is also considered as one of the major constraints. With the development of low-cost smart sensor nodes [12], CR technology is also regarded as an applicable alternative in WSNs to provide multitask support and efficient delivery of bursty traffic.

\subsection{Hybrid MAC Protocols in DCRNs}

Spectrum efficiency is the ultimate objective in DCRNs. To this end, many studies have been conducted. Generally, MAC protocols in DCRNs are divided into three categories: random access, time-slotted, and hybrid [9][10]. Recently, the hybrid protocol has been considered the most suitable for CRNs because it tries to achieve a tradeoff between the random access and time-slotted protocols [14]. 
The representative hybrid protocols are opportunistic MAC (O-MAC) [15], opportunistic multi-channel MAC (OMC-MAC) [16], and multi-channel MAC (MC-MAC) [17]. These MAC protocols are mostly categorized as spectrum overlay dynamic spectrum access (DSA) [10]. In these kinds of protocols, spectrum sensing is the most important function because SUs should opportunistically access the spectrum bands only when they are not occupied by primary users (PUs). OMC-MAC [16] and MC-MAC [17] allow each SU to employ local spectrum sensing to find available spectrum bands, but O-MAC [15] uses cooperative spectrum sensing to alleviate sensing errors. To identify which channels are idle, the random sensing policy (RSP) and the negotiation-based sensing policy (NSP) are developed in O-MAC [15]. However, because collision probabilities are used by more than two SUs to transmit their beacons in the reporting phase, and SUs use $p$-persistent carrier sense multiple access (CSMA) protocols to reserve data channels for their data transmission, throughput can be degraded.

For the exchange of control packets, MAC protocols apply dedicated control channels [23][24], which is an approach that allows all SUs to listen to the control channel at all times, even during data transmission. It is well known that this approach is suitable for hybrid protocols in DCRNs. In O-MAC [15] and MC-MAC [17], each SU is equipped with multiple transceivers to exchange control messages and transfer data simultaneously, while a single transceiver is deployed in OMC-MAC [16]. Accordingly, the throughput decreases because the residual time except for sensing time is used for data transmission. Although MC-MAC [17] uses a dedicated control channel, the throughput is degraded because the data transmission can be carried out after the sensing phase within a timeslot.

In recent years, energy efficiency has become an issue in CRNs; however, few relevant studies have been conducted. As in the power saving mechanisms (PSM) in the IEEE 802.11 DCF [25], in Multichannel MAC protocol for Cognitive Radio (MMAC-CR) [18], time is divided into an ad hoc traffic indication message (ATIM) window and a data window. The control packets in DCRNs are transferred in the ATIM windows, whereas the data packets are transferred in the data windows. Similar to MMAC-CR [18], the energy-efficient CR MAC for QoS provisioning (ECRQ-MAC) [19] is proposed. These protocols are less prone to the common control channel (CCC) bottleneck problem because only one handshake on the CCC is needed per connection during the beacon interval. Little control information is required compared to other protocols, where one handshake per sent packet is needed. On the other hand, these protocols wait until the end of the ATIM window to send and receive data packets. Therefore, the channel bandwidth of all data channels in the ATIM window is wasted, which leads to more delay and lower throughput [20]. In [21], in order to extend the total network lifetime, a cooperative schedule for the on/off time of each sensor node is proposed. However, the assumptions are unpractical in which an abundant number of sensor nodes is required and synchronization among them is well performed. As in [21], for the reduction of energy consumption, a preemptive opportunistic MAC, named PO-MAC [22], in DCRNs is proposed. The proposed scheme has introduced a cooperative sensing scheme to reduce the energy consumption. However, they have not conducted a performance evaluation for energy efficiency, such as network lifetime.

\section{System Model}

In this paper, we consider a CR-enabled sensor network (CRSN) with $N_{p u}$ data channels licensed by PUs. A SU can access one of those PU channels when no PU activity is detected over the channel. We will assume that the PU activity on a PU channel is characterized by an ON-OFF source model alternating between ON (busy) and OFF (idle) states, which has been 
widely used in numerous existing works [9]. To efficiently use available spectrum resources, the dedicated control channel approach [23][24] is applied to perform the control signaling and data transmission simultaneously. Hence, it is assumed that there is a single control channel utilized by the SUs in the network. Then, an SU is equipped with two half-duplex transceivers: a control transceiver and a data transceiver. This can be achieved by keeping the control transceiver continuously tuned to the control messages, while the data transceiver is being used for data communications. Because an SU can be aware of the exchange of control messages among neighboring SUs, the multi-channel hidden terminal problem (MHTP) becomes easier to handle with multiple transceivers. With advancements in micro-electromechanical system (MEMS) technology [26], it is sufficiently possible to equip multiple transceivers in a wireless sensor node.

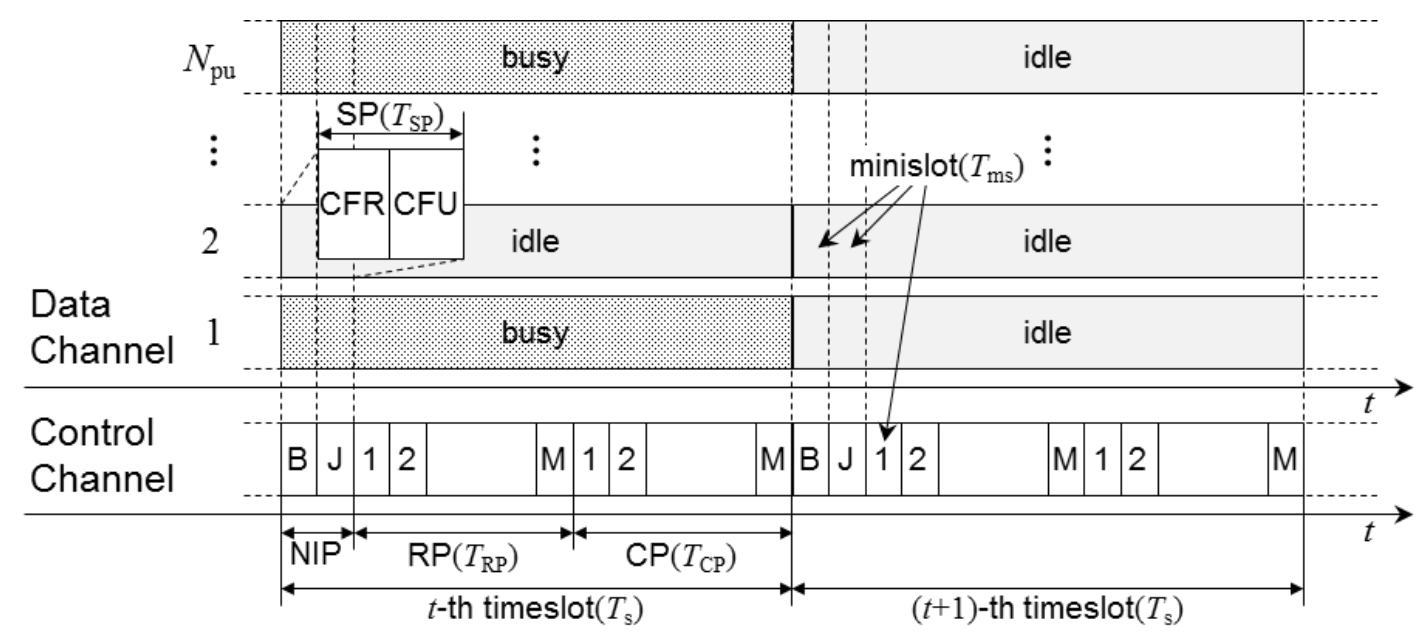

Fig. 1. Time structure for ENC-MAC in a CRSN.

Fig. 1 shows the time structure for ENC-MAC in the CRSN. The time axis is divided into a number of periodic timeslots. All timeslots of the control channel have the same time length, $T_{s}$, as those of the data channels, and the timeslots of the control channel and the data channels are synchronized by broadcasting a beacon message at the beginning of every timeslot over the control channel. The details of the beacon message are described in Section 4.1. Each timeslot begins with the sensing phase (SP), which lasts for $T_{S P}$. Note that the sensing phase is the dedicated time for sensing the assigned channels. That is, an SU uses the data transceiver to check whether the PUs exist over a certain data channel or not. Then, what happens in the control channel during this time is known as the network initialization phase (NIP), which is detailed in Section 4.1. A timeslot is further divided into several minislots with time length $T_{m s}$, and the number of minislots in a timeslot can be varied by the number of data channels and the number of SUs in the network. Further, the length of a minislot is enough to confirm the state of one of the data channels or to exchange control messages for network joining and channel contention. Again, the sensing phase has two minislots: one is for an SU to sense a channel for report (CFR), and the other is for the SU to sense a channel for use (CFU). The number of minislots in the sensing phase should be as few as possible because the frequency of sensing directly affects transmission capabilities [27]. In this method, in order to detect the PUs' activities, the SUs are assumed to use an energy detection mechanism, which is widely adopted in CRNs [28][29]. The spectrum sensor of each SU detects the presence of PU signals by performing the binary hypothesis test as follows: 


$$
H_{0}: S_{i}(t)=0(\text { idle }), \quad \text { and } H_{1}: S_{i}(t)=1 \text { (busy), }
$$

where $S_{i}(t) \in\{0$ (idle), 1 (busy) $\}$ denotes the PU's occupancy state of channel $i\left(1 \leq i \leq N_{\text {pu }}\right)$ at the $t$-th timeslot $(t \geq 0)$. The details of the sensing phase are explained in Section 4.2.

The sensing results for a CFR in the sensing phase are reported in the reporting phase over a control channel, as detailed in Section 4.3. At the end of the reporting phase, the SUs form the available channel list (ACL). Thus, with the ACL, the SUs contend with each other and reserve their data channels in the contention phase, which is described in Section 4.4. For simplicity, it was designed that both the reporting phase and contention phase have the same number of minislots $M$, where $M \geq N_{\mathrm{pu}}$. Thus, the length of a timeslot for all the phases is given by:

$$
T_{s} \geq T_{S P}+T_{R P}+T_{C P}=(2+2 M) \cdot T_{m s} \text { where } \quad M \geq N_{p u} .
$$

Note that the data channels reserved in the current timeslot are not used right away, but they can be used in the following timeslot. For instance, the data channels reserved in the $t$-th timeslot can be used in $(t+1)$-th timeslot to transmit data packets and acknowledgements. Because the length of a timeslot can be varied according to system parameters, multiple packet transmission and/or concatenation schemes are required, but this falls outside the scope of this paper.

\section{ENC-MAC: Energy-efficient Non-overlapping Channel MAC}

\subsection{Network Initialization Phase}

Network initialization is the process whereby additional SUs join the network and their timing is synchronized with the existing SUs. For this process, we define two types of SUs according to their roles: a master SU (MSU) and a normal SU (NSU). In the operation of ENC-MAC, one MSU is required to send the beacon message at the beginning of each timeslot, i.e., the 'B' minislot as shown in Fig. 1. The procedure for self-determination of the MSU is as follows: An SU joining the network first attempts to receive beacon messages.

If it does not receive any beacon messages for $K$ consecutive timeslots, e.g., $K=3$, it is concluded that it is the first SU in the network [22]. Therefore, the proposed scheme makes it become the MSU in the network and proceeds to send beacon messages, including a timestamp, periodically. If SUs that want to join the network receive the beacon message from the MSU, they are able to synchronize their times with both the MSU's and other existing SUs' times by using the timestamp contained in the beacon message.

After the synchronization process, SUs joining the network compete for the control channel at the second minislot of the timeslot, i.e., the 'J' minislot as shown in Fig. 1. The competition process is based on the backoff mechanism that is similar to the IEEE 802.11 DCF [25], and its details are described in Section 4.4. If an SU wins the contention successfully, it broadcasts a JOIN message over the control channel using the ' $\mathrm{J}$ ' minislot. In response to the JOIN message, the MSU may transmit a beacon message over the control channel. In order to let the SU know the CFR and maintain the SIL, the beacon message includes the MAC address and index of the joining SU ( $<$ MAC addr $>,<$ SU index $>$ ), and the number of SUs in the network in the $t$-th timeslot $\left.\left(<N_{s u}(t)\right\rangle\right)$. The MSU maintains the assignment of indices for joining SUs. The index of each joining SU is assigned by the MSU and the earlier the SU joins the network, the lower 
index it has. As a result, the existing SUs can acknowledge the $<\mathrm{SU}$ index $>$ of a new SU, and can maintain their SUs' identifier list (SIL).

The case when the MSU disappears or fails in the network should be considered. If no beacon messages are received from the MSU for $K$ consecutive timeslots, the SUs recognize the absence or failure of the MSU. Then, the SU with the lowest index among existing SUs becomes a new MSU, thereafter periodically sending beacon messages. It is noted that SUs' indices have been assigned by the former MSU, and each SU maintains its own SIL by hearing the exchange of JOIN and CFR messages between SUs and the MSU. That is, with the SIL, the SU can acknowledge whether it has the lowest index value or not.

\subsection{Sensing Phase}

Given $N_{p u}$ and $N_{s u}(t)$, each SU with a different index can identify the $i$-th CFR $\left(1 \leq i \leq N_{p u}\right)$ to sense at the first minislot of the sensing phase as shown in Fig. 1. If $N_{s u}(t) \geq N_{p u}$, in order to sense and report data channels without omission, the index of the SU that senses the $i$-th CFR solely at the $t$-th timeslot, i.e., $I(t, i)$, is obtained by:

$$
\left.I(t, i)=\bmod \left(\left(N_{p u} \cdot t\right)+(i-1)\right), N_{s u}(t)\right)+1,
$$

where $\bmod (a, b)$ is the modulo operation that finds the remainder after division of number $a$ by number $b$. The reason that a PU channel is sensed and reported by the different SUs with their local conditions at every timeslot, as shown in Fig. 2 (a), is to increase the reliability of the sensing results. Otherwise, i.e., when $N_{s u}(t)<N_{p u}, I(t, i)$ is given by:

$$
I(t, i)=\bmod \left(((i-1)-t), N_{p u}\right)+1 .
$$

Fig. 2 (a) and (b) show examples of $I(t, i)$, when $N_{s u}(t) \geq N_{p u}$ and $N_{s u}(t)<N_{p u}$, respectively. As shown in Fig. 2, only the SUs indicated by $I(t, i)$ are requested to sense data channels. In Fig. 2 (b), two data channels cannot be sensed by existing SUs because one SU can only sense each CFR at the timeslot. Accordingly, when $M \geq N_{p u}$, more than two minislots are not used in the reporting phase and the contention phase. However, this is a natural phenomenon by design of the proposed scheme.

Owing to one SU's exclusive sensing and reporting on each data channel, ENC-MAC can resolve the collision problem that occurs when multiple SUs sense and report on the same channel, as is the case with O-MAC [15]. Further, the proposed scheme can reduce the waste of energy that happens when one SU should consume a considerable amount of time and energy to sense and report all data channels, as in OMC-MAC [16].

\begin{tabular}{|c|c|c|c|c|c|c|c|c|}
\hline $\boldsymbol{t}$ & $\mathbf{0}$ & $\mathbf{1}$ & $\mathbf{2}$ & $\mathbf{3}$ & $\mathbf{4}$ & $\mathbf{5}$ & $\mathbf{6}$ & $\ldots$ \\
\hline $\mathbf{1}$ & 1 & 6 & 11 & 3 & 8 & 13 & 5 & $\ldots$ \\
\hline $\mathbf{2}$ & 2 & 7 & 12 & 4 & 9 & 1 & 6 & $\ldots$ \\
\hline $\mathbf{3}$ & 3 & 8 & 13 & 5 & 10 & 2 & 7 & $\ldots$ \\
\hline $\mathbf{4}$ & 4 & 9 & 1 & 6 & 11 & 3 & 8 & $\ldots$ \\
\hline $\mathbf{5}$ & 5 & 10 & 2 & 7 & 12 & 4 & 9 & $\ldots$ \\
\hline
\end{tabular}

(a) $N_{p u}=5$ and $N_{s u}(t)=13\left(N_{s u}(t) \geq N_{p u}\right)$

\begin{tabular}{|c|c|c|c|c|c|c|c|c|}
\hline $\boldsymbol{t}$ & $\mathbf{0}$ & $\mathbf{1}$ & $\mathbf{2}$ & $\mathbf{3}$ & $\mathbf{4}$ & $\mathbf{5}$ & $\mathbf{6}$ & $\ldots$ \\
\hline $\mathbf{1}$ & 1 & - & - & 3 & 2 & 1 & - & $\ldots$ \\
\hline $\mathbf{2}$ & 2 & 1 & - & - & 3 & 2 & 1 & $\ldots$ \\
\hline $\mathbf{3}$ & 3 & 2 & 1 & - & - & 3 & 2 & $\ldots$ \\
\hline $\mathbf{4}$ & - & 3 & 2 & 1 & - & - & 3 & $\ldots$ \\
\hline $\mathbf{5}$ & - & - & 3 & 2 & 1 & - & - & $\ldots$ \\
\hline
\end{tabular}

(b) $N_{p u}=5$ and $N_{s u}(t)=3\left(N_{s u}(t)<N_{p u}\right)$

Fig. 2. Examples of $I(t, i)$, the SUs that sense the $i$-th data channel solely at the $t$-th timeslot. 


\subsection{Reporting Phase}

An SU cannot accurately know the states of all data channels by itself because it is designed such that it can sense only one data channel, i.e., CFR, determined by Eqs. (3) and (4), at every timeslot. Thus, in this method, the cooperative sensing technique [29] is essential to enable the SUs to have a large picture of all states of data channels through their cooperation.

The report message, which is used to report the sensing results, consists of three main fields: < SU index >, < CFR >, and < result $>$. The first two fields denote the SU's identifier and its data channel for reporting, i.e., CFR, respectively. The last field is represented by one bit: 0 (idle), or 1 (busy) as given in Eq. (1). Considering ordinary CR operational environments with the maximum number of available channels and manageable SUs, the report message can consist of two bytes. When the IEEE 802.11 [25] and IEEE 802.22 [30] standards are considered to be the physical medium, the minislot duration of $500 \mu$ s that is defined by the specifications is used. The length of the minislot is enough to deliver all kinds of control packets used in our proposed scheme, as well as to identify states of data channels.

Once $I(t, i)$ have been determined using Eqs. (3) and (4), the SU corresponding to $I(t, i)$ begins to sense the $i$-th CFR to report its state by using the report message at the $i$-th minislot in the reporting phase at the $t$-th timeslot. Then, whenever an SU receives the message from others, it updates its ACL. Then, the SUs use their up-to-date ACL to contend for and reserve available data channels in the contention phase.

\subsection{Contention Phase and Data Transmission Phase}

In this section, the process for assigning channels to transmit data packets, i.e., CFUs (refer to Section 3), to the SUs is introduced. The SUs maintain the available channel list (ACL) and the number of available data channels by means of cooperative sensing with others in the reporting phase as described in Section 4.3. Then, in the contention phase, they contend for and reserve idle data channels only when an SU wants to transmit its data packet. The contention phase consists of $M$ minislots as shown in Fig. 1, and $N_{a v}$ or up to $M$ minislots can be used for the SUs that have data packets to contend for the reservation of idle data channels.

For the channel reservation together with collision resolution of control messages in the contention phase, the request-to-send (RTS)/clear-to-send (CTS) exchange with backoff time is used, which is based on the IEEE 802.11 DCF [25]. Additionally, the RTS and CTS messages are modified to embed a $<\mathrm{CFU}>$ field ( 1 byte) to reserve the CFU between the sender and receiver SUs and to inform the remaining SUs of the CFU by overhearing the messages.

The overall channel contention process using the RTS/CTS exchange proceeds as described in the remainder of this section. The SUs that want to transmit data packets wait for random backoff intervals at the beginning of each minislot in the contention phase. The minislot can be used only if the corresponding channel is idle as identified by the ACL. After their backoff intervals, the winning SU transmits its RTS message containing the selected CFU to its intended receiver SU. If the receiver SU gets the RTS message, and the contained CFU is confirmed to be idle from its ACL, it responds with a CTS message with the same CFU. Then, the $\mathrm{CFU}$ is reserved for the sender and receiver SUs. The remaining SUs also can update their ACLs by removing the CFU after overhearing the RTS/CTS exchange. In the same way, the remaining SUs continue to contend for available data channels with the updated ACL. Thus, the MHTP can be resolved by the exchange of control messages in the contention phase over the control channel. This process may continue until there are no more SUs that want to transmit data packets or no more available data channels in the network. 
Next, the winner SU senses the selected CFU at the second minislot in the sensing phase of the next following timeslot as shown in Fig. 1. This is because the state of the selected CFU at the next following timeslot may be changed such as from idle to busy or vice versa. If the CFU is still idle, the SU can transmit its data packet over the CFU. Otherwise, that is, if the state of the CFU is changed from idle to busy, the SU is not able to transmit its data packet and should carry out the contention process again in the contention phase at the timeslot.

\section{Analytical Model}

\subsection{Throughput for Saturation Network}

In order to analyze the saturation throughput as a major performance measure to evaluate the proposed scheme, we assume that all SUs always have data packets to transmit; at an SU, a successfully transmitted data packet is replaced immediately by another data packet that needs to be transmitted. This is called the infinite backlog model [31].

We modeled each data channel as an ON/OFF source that independently alternates between busy and idle states. Let $\alpha_{i}$ and $\beta_{i}$ be the probabilities that the $i$-th data channel remains in its busy and idle state, respectively. Then, the $i$-th data channel utilization $\gamma_{i}$ occupied by a PU is given by:

$$
\gamma_{i}=\lim _{T \rightarrow \infty} \frac{1}{T} \sum_{t=0}^{T} S_{i}(t)=\frac{\alpha_{i}}{\alpha_{i}+\beta_{i}}
$$

Suppose that there are $N_{p u}$ data channels with the same channel utilization $\gamma$ obtained by:

$$
\gamma=\gamma_{i}=\gamma_{j} \text { where } \forall 1 \leq i, j \leq N_{p u} \text { and } i \neq j .
$$

Let $P_{\text {succ }}$ be the probability that an idle data channel is successfully reserved by one SU during a minislot in the contention phase. From the result of [32], this is written as:

$$
P_{\text {succ }}=\frac{N_{p u} \cdot p_{r} \cdot\left(1-p_{r}\right)^{N_{p u}-1}}{1-\left(1-p_{r}\right)^{N_{p u}}},
$$

where $p_{r}$ is the probability that an SU transmits its RTS message in a backoff slot time during a minislot in the contention phase, which is detailed in [32], as a function of the contention window size and the conditional collision probability.

Let $N_{\text {avail }}(t)$ be the number of idle data channels at the $t$-th timeslot. Because the states among $N_{p u}$ channels are independent, and the active probability of the channel is $\gamma$, we obtain the probability that $N_{\text {avail }}(t)=k$, where $1 \leq k \leq N_{\mathrm{pu}}$, as follows:

$$
\operatorname{Pr}\left\{N_{\text {avail }}(t)=k\right\}=\left(\begin{array}{c}
N_{p u} \\
k
\end{array}\right) \gamma^{N_{p u}-k} \cdot(1-\gamma)^{k}
$$

Let $M_{s u}(t)$ be the number of minislots reserved by SUs at the $t$-th timeslot. Then, the probability that $M_{s u}(t)=m$, where $m=1,2, \ldots, M$, is given by: 


$$
\operatorname{Pr}\left\{M_{s u}(t)=m\right\}=\left(\begin{array}{c}
M \\
m
\end{array}\right) P_{\text {succ }}^{m} \cdot\left(1-P_{\text {succ }}\right)^{M-m}
$$

Let $N_{a v}$ be the average number of idle data channels, and $N_{r s v}$ be the average number of minislots that SUs can reserve for those idle channels. Since $N_{\text {avail }}(t)$ and $M_{s u}(t)$ follow binomial distribution with probabilities $1-\gamma$ and $P_{\text {succ }}$, respectively, from Eqs. (8) and (9), we have

$$
\begin{gathered}
N_{a v}=\sum_{k=0}^{N_{p u}} k \cdot \operatorname{Pr}\left\{N_{\text {avail }}(t)=k\right\}=N_{p u} \cdot(1-\gamma) \\
N_{r s v}=\sum_{m=0}^{M} m \cdot \operatorname{Pr}\left\{M_{s u}(t)=m\right\}=M \cdot P_{\text {succ }}
\end{gathered}
$$

It is noted that $N_{a v}$ is the average number of idle channels that SUs can acknowledge during the report phase. However, as a result of the contention for those idle channels, the number of actually reserved channels, $N_{r s v}$, may be equal to or less than $N_{a v}$. In addition, as mentioned earlier, although a SU reserves an idle data channel in contention phase during a timeslot, it can transmit its data only when the reserved data channel is also idle at the next timeslot. Thus, when we let $N_{\text {avg }}$ be the average number of SUs that can transmit their data over their reserved channels at the next timeslot, it can be written

$$
N_{a v g}=\min \left(N_{a v}, N_{r s v}\right) \cdot \alpha
$$

where $\alpha$ is the probability that the reserved channel state remains idle at the next timeslot.

Let $R_{i}$ be the data rate of the $i$-th data channel, where $1 \leq i \leq N_{\mathrm{pu}}$, for the SUs in the network. Without loss of generality, we assume that all $N_{p u}$ data channels have the same bandwidth, that is, $R=R_{i}=R_{j}$, where $\forall 1 \leq i, j \leq N_{p u}$ and $i \neq j$. Finally, the saturation throughput $S$ for the SUs is obtained by:

$$
S=N_{a v g} \cdot R \cdot \frac{\left(T_{s}-T_{S P}\right)}{T_{s}},
$$

where the factor $\left(T_{s}-T_{S P}\right) / T_{s}$ is originated from the fact that the data transmission starts immediately after the sensing phase and continues for a period of $\left(T_{s}-T_{S P}\right)$ in every timeslot.

\subsection{Average Packet Delay for Non-Saturation Network}

In order to analyze the average packet delay for non-saturation networks, it is required that a network should be modeled as a system. Thus, we construct the system that has a single queue with a time-varying capacity. Suppose that there are $N_{s u}$ SUs in the network with the same packet arrival rate $\lambda$ :

$$
\lambda=\lambda_{i}=\lambda_{j} \text { where } \forall 1 \leq i, j \leq N_{s u} \text { and } i \neq j
$$

The packet arrivals to the queue can be modeled as the aggregated arrivals from a CRN from which the SUs are opportunistically accessing idle data channels. Then, the aggregated 
channel capacity over time can be seen by the aggregated packet arrivals in the network through the operation of the proposed scheme.

When the arrival rate of data packets of each SU is Poisson distribution, the aggregated arrivals of $N_{s u}$ SUs to the idle data channels in the network are also Poisson with $N_{s u} \cdot \lambda$ as follows:

$$
N_{s u} \cdot \lambda=\sum_{i=1}^{N_{s u}} \lambda_{i} .
$$

The server capacity of this queue is dependent on the distribution of the PUs' occupancy and is assumed to be generally distributed. Thus, the network can be modeled as an $M / G / 1$ queuing model [33][34] to investigate the average packet delay. Let $N_{s r v}$ be the average number of data packets delivered within a timeslot. Here, we assume that a winner SU can transmit one data packet at once over the CFU during a timeslot. Then, since the average number of SUs that can transmit during a timeslot is given $N_{a v g}$, we have $N_{s r v}=N_{a v g}$. Accordingly, we have the service rate of the network as follows:

$$
\mu=\frac{N_{a v g}}{T_{s}}
$$

Let $L_{q}$ be the average number of data packets in the queue of the system. Then, we have

$$
L_{q}=\frac{\lambda^{2} E\left[S^{2}\right]}{2(1-\rho)},
$$

where $\rho$ is the traffic intensity, i.e., $\rho=\lambda / \mu$, where $\rho<1$. According to Little's law $(L=\lambda \cdot W)$, which is broadly applied to queuing systems, the average packet delay in the network $W_{s}$ is given by:

$$
W_{s}=\frac{L_{q}}{\lambda}+E[S],
$$

where $E[S]=1 / \mu$ stands for the service time of the network.

\subsection{Network Lifetime}

In order to analyze the energy efficiency of the proposed scheme, we applied the network lifetime as a performance metric to measure the time duration before a given portion of wireless sensor nodes run out of energy. The time structure proposed in this paper is similar to that of the IEEE 802.11 power saving mechanism (PSM) [25].

Compared with the PSM of IEEE 802.11, the difference is that the CR-based MAC protocols consider the sensing for data channels, which is the most important function to affect the overall performance in accordance with its sensing results. The power consumption is classified into four categories: transmit, receive, and idle modes and doze state. In the proposed scheme, in which energy detection is deployed for fast sensing [30], the power consumption of the sensing is assumed to be the same as that of the idle mode. 
Let $E_{S P}$ be the average energy measured in Joules per a timeslot for sensing channels, then we have

$$
E_{S P}=e_{i d l e} \cdot T_{S P}
$$

where $e_{i d l e}$ denotes the electric power required for the idle mode for one second in watts. Accordingly, the spectrum sensing technique is of importance because it determines $T_{S P}$.

The contention phase is similar to the ATIM window in the PSM of IEEE 802.11. Thus, this phase nearly operates in receive mode except for transmitting the control messages. According to the roles of sensing nodes, i.e., sender, receiver, and neighbor, the energy measured in Joules per a timeslot for the contention phase is as follows:

$$
\left\{\begin{array}{l}
E_{C P, \text { sender }}=e_{r x} \cdot\left(T_{C P}-T_{R T S}\right)+e_{t x} \cdot T_{R T S} \\
E_{C P, \text { receiver }}=e_{r x} \cdot\left(T_{C P}-T_{C T S}\right)+e_{t x} \cdot T_{C T S}, \\
E_{C P, \text { neighbor }}=e_{r x} \cdot T_{C P}
\end{array}\right.
$$

where $e_{r x}$ and $e_{t x}$ are the electric power of the receive and transmit modes for one second in watts, respectively, and $T_{R T S}$ and $T_{C T S}$ are the times for transmitting RTS and CTS messages in the network, respectively. The neighbors that overhear the control messages are operated in the receive mode. Then, the average energy consumption measured in Joules per a timeslot for contention phase $E_{C P}$ is written as:

$$
E_{C P}=\frac{N_{\text {avg }} \cdot\left(E_{C P, \text { sender }}+E_{C P, \text { receiver }}\right)+\left(N_{s u}-2 \cdot N_{\text {avg }}\right) \times E_{C P, \text { neighbor }}}{N_{s u}} .
$$

where $2 \cdot N_{\text {avg }}$ stands for the number of contention winners and their intended receivers for $N_{\text {avg }}$ data channels in the network.

Lastly, the data transmission phase is analogous to the communication window in the PSM of IEEE 802.11. For simplicity, data transfer without acknowledgement is considered. According to the roles of sensing nodes, the energy measured in Joules per a timeslot for the data transmission phase is as follows:

$$
\left\{\begin{array}{l}
E_{D T P, \text { sender }}=e_{t x} \cdot T_{D T P} \\
E_{D T P, \text { receiver }}=e_{r x} \cdot T_{D T P} \\
E_{D T P, \text { neighbor }}=e_{d o z e} \cdot T_{D T P}
\end{array}\right.
$$

where $e_{\text {doze }}$ is the electric power of doze for one second in watts. The neighbors that do not receive data packets enter the doze state. This reduces energy wastage due to idle listening [25]. Then, we have the average energy measured in Joules per a timeslot for data transmission phase $E_{D T P}$ as follows:

$$
E_{D T P}=\frac{N_{a v g} \cdot\left(E_{D T P, \text { sender }}+E_{D T P, \text { receiver }}\right)+\left(N_{s u}-2 \cdot N_{\text {avg }}\right) \times E_{D T P, \text { neighbor }}}{N_{s u}} .
$$

Using Eqs. (19), (21), and (23), the network lifetime $T_{\text {life }}$, with respect to the initial energy in watts $E_{\text {init }}$, is obtained by: 


$$
T_{\text {life }}=\left\lceil\frac{E_{\text {init }}}{\left(E_{S P}+E_{C P}+E_{D T P}\right)} \cdot \frac{1}{T_{s}}\right\rceil,
$$

where $1 / T_{s}$ denotes the number of timeslots in one second.

\section{Performance Evaluation}

Table 1. Simulation parameters used for ENC-MAC protocol.

\begin{tabular}{|l|l|r|}
\hline Parameter & Description & Value \\
\hline \hline$e_{\text {doze }}$ & Energy power of doze state & $0.045 \mathrm{~W}$ \\
\hline$e_{i d l e}$ & Energy power of idle mode & $1.15 \mathrm{~W}$ \\
\hline$e_{r x}$ & Energy power of receive mode & $1.4 \mathrm{~W}$ \\
\hline$e_{t x}$ & Energy power of transmit mode & $1.65 \mathrm{~W}$ \\
\hline$L_{C T S}$ & Clear-to-send size & $120 \mathrm{bits}$ \\
\hline$L_{R T S}$ & Request-to-send size & $168 \mathrm{bits}$ \\
\hline$N_{p u}$ & Number of data channels & 10 \\
\hline$N_{s u}$ & Number of secondary users & 20 \\
\hline$R$ & Data rate of data channels & $1 \mathrm{Mbps}$ \\
\hline$T_{m s}$ & Length of minislot & $500 \mu \mathrm{s}$ \\
\hline$T_{s}$ & Length of timeslot & $20 \mathrm{~ms}$ \\
\hline$T_{s l o t}$ & Length of backoff window & $20 \mu \mathrm{s}$ \\
\hline
\end{tabular}

For the analysis and simulation of ENC-MAC, MATLAB was utilized as the performance evaluation tool. The parameters for the performance evaluation are summarized in Table 1. Unless otherwise specified, the following parameters will be used in the plots in this section. In order to construct the evaluation environment, we set the number of data channels occupied by the PUs such that each channel had a data rate of 1 Mbps. Considering IEEE 802.11 [25] as the physical medium, $T_{m s}, T_{s}$, and $T_{\text {slot }}$ were set to $500 \mu \mathrm{s}, 20 \mathrm{~ms}$, and $20 \mu \mathrm{s}$, respectively. The length of RTS and CTS messages were set to 168 and 120 bits, respectively. For the energy model, we used $1.65 \mathrm{~W}, 1.4 \mathrm{~W}, 1.15 \mathrm{~W}$, and $0.045 \mathrm{~W}$ as values of energy power [19] consumed by the wireless network interface in the transmit, receive, and idle modes and the doze state, respectively.

Fig. 3 represents how the saturation throughput for ENC-MAC, OMC-MAC, and O-MAC was affected by the degree of PU channel utilization, i.e., $\gamma=0.1 \sim 0.9$. The reason that the O-MAC [15] and OMC-MAC [16] protocols were used as the comparison schemes is that ENC-MAC is a hybrid protocol that is operated with multiple data channels and a dedicated control channel. Additionally, ENC-MAC is inspired from O-MAC, which is the latest of the hybrid protocols in these kinds of networks. The same parameters in [15] and [16] were utilized to obtain the throughput values for O-MAC and OMC-MAC, respectively. For the $p$-persistent CSMA operation of O-MAC, we set $p$ to $1 / N_{s u}$, at which O-MAC shows its maximum throughput. 


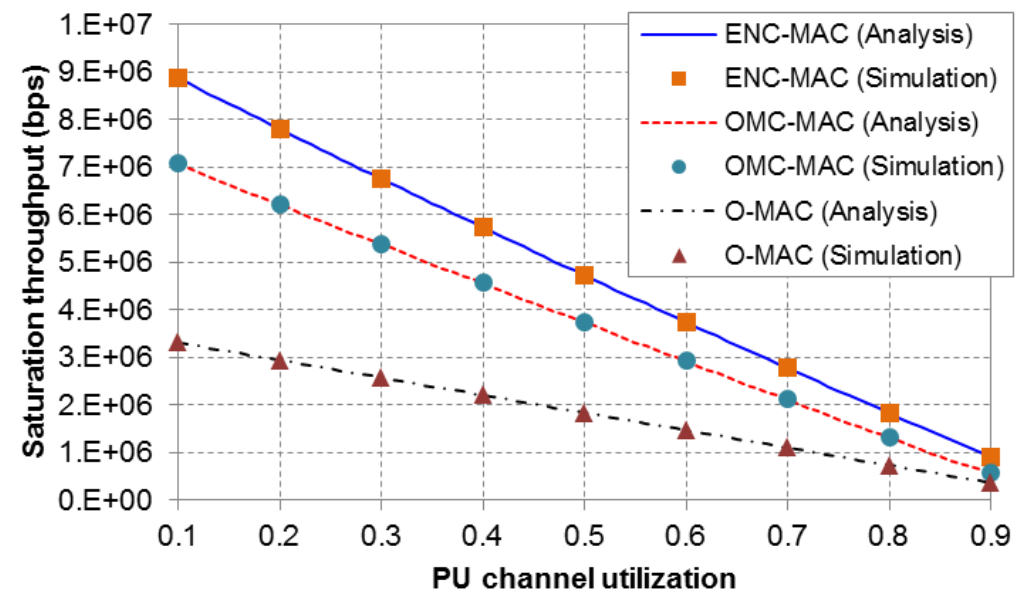

Fig. 3. Saturation throughput versus PU channel utilization for ENC-MAC, O-MAC, and OMC-MAC, where $N_{p u}=10$ and $N_{s u}=2 \cdot N_{p u}$.

As expected, ENC-MAC achieved a higher saturation throughput than both O-MAC and OMC-MAC. Compared to OMC-MAC, ENC-MAC was not affected by the number of data channels because the timeslot - except for sensing time-was fully utilized for the data transfer, which is because of the control channel. In addition, ENC-MAC allocates minislots in the reporting phase to the SUs in a non-overlapping manner. Therefore, the SUs in ENC-MAC can perceive the state of data channels unoccupied by PUs without collisions, which is in contrast with O-MAC using $p$-persistent CSMA to report the channel states. The utilization of $p$-persistent CSMA in O-MAC resulted in a needless delay to access available data channels. Thus, O-MAC showed the worst saturation throughput. In OMC-MAC, the exponential backoff algorithm is applied for RTS/CTS exchange to reserve vacant data channels, and thus it showed much better throughput than O-MAC. Meanwhile, a timeslot in OMC-MAC consists of three phases: sensing, contention, and transmission. The duration of the former two phases cannot be utilized for data transmission because these three phases are executed in a sequential way within a timeslot. As a result, it is obvious that ENC-MAC outperformed both $\mathrm{O}-\mathrm{MAC}$ and OMC-MAC.

Fig. 4 illustrates the saturation throughput for the analysis and simulation as the number of SUs increases when the PU channel utilization is 0.3 and 0.6. In addition, this graph verifies that the maximum throughput can be achieved regardless of the PU channel utilization. When $N_{s u}<N_{p u}$, the saturation throughput for all cases increased linearly as $N_{s u}$ increased. However, because a data transceiver equipped in an SU is used for a single data channel only, the throughput for all cases became saturated after $N_{s u} \geq N_{p u}$.

All our numerical results were validated by simulation. In order to show this, Fig. 5 plots the average packet delay versus PU channel utilization, where $N_{p u}=10$ and 15 . It is observed in Fig. 5 that the average number of packets increases linearly as PU channel utilization increases and the number of data channels decreases, and the resources are scarce because the SUs take more time to find available data channels in the network. 


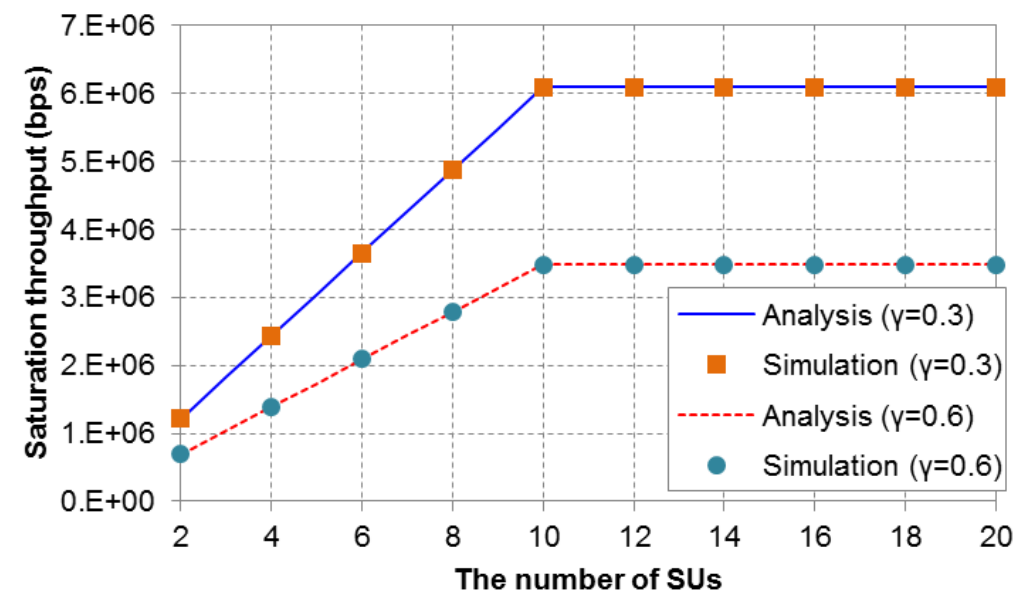

Fig. 4. Saturation throughput versus the number of SUs for $\gamma=0.3$ and 0.6 and $N_{p u}=10$.

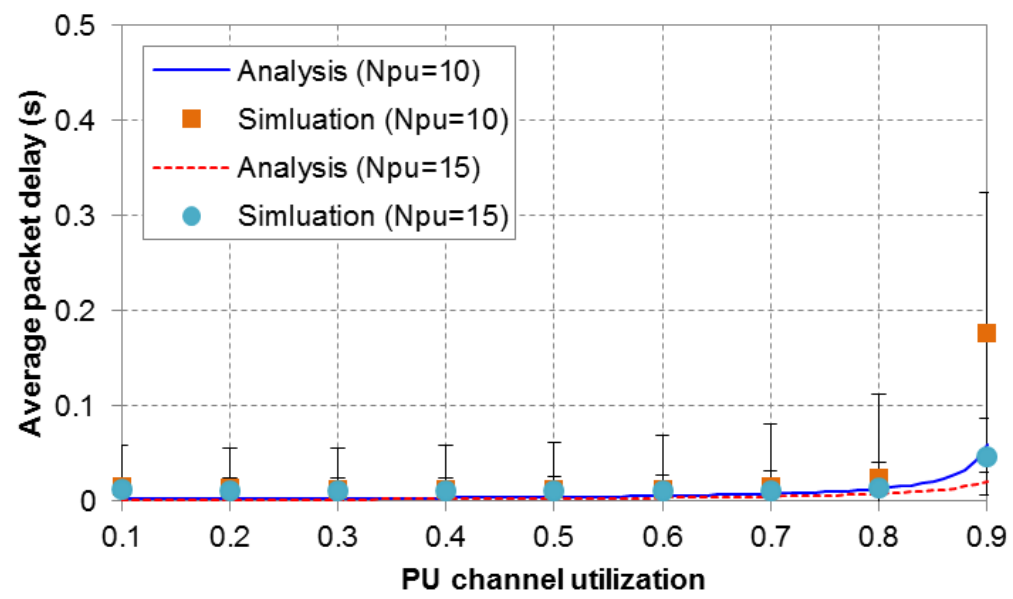

Fig. 5. Analytical and simulation results for average packet delay versus PU channel utilization for $N_{p u}$ $=10$ and 15 .

Fig. 6 depicts the analytical results for the average packet delay versus the utilization of channels occupied by PUs where the following parameters are varied: (a) $N_{p u}=10-18$ by 1 , $N_{s u}=10$, and $\lambda=5$, and (b) $N_{p u}=10, N_{s u}=1-9$ by 1 , and $\lambda=5-45$ by 5 [refer to Eq. (15)]. Note that the traffic intensity is always lower than one, i.e., $\rho<1$, in Fig. 6. In the plots of Fig. 6, a 3D representation is used in order to simultaneously visualize the effect of (a) and (b). It is shown in Fig. 6 (a) and (b) that the average packet delay increases when the PU utilization increases. When $\gamma \geq 0.7$, the situation intensifies. Fig. 6 (a) illustrates that as the number of PU channels increases, the packet delay decreases because traffic intensity gets relatively lower. It is shown in Fig. $\mathbf{6}$ (b) that the impact on the number of SUs is insignificant because even if the number of SUs is increased, the traffic intensity is less than one, i.e., $\rho<1$. 


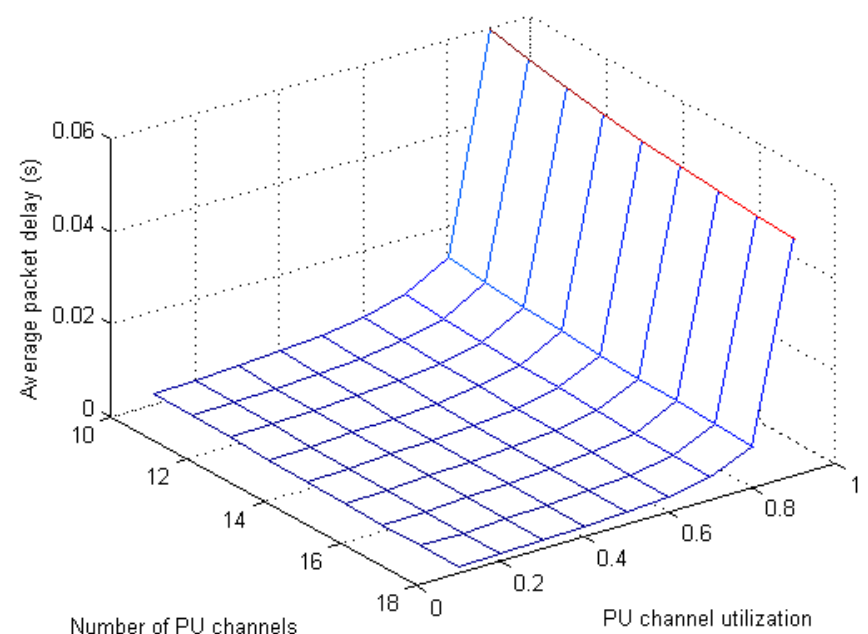

(a) $N_{p u}=10$ to 18 by $1, N_{s u}=10$, and $\lambda=5$

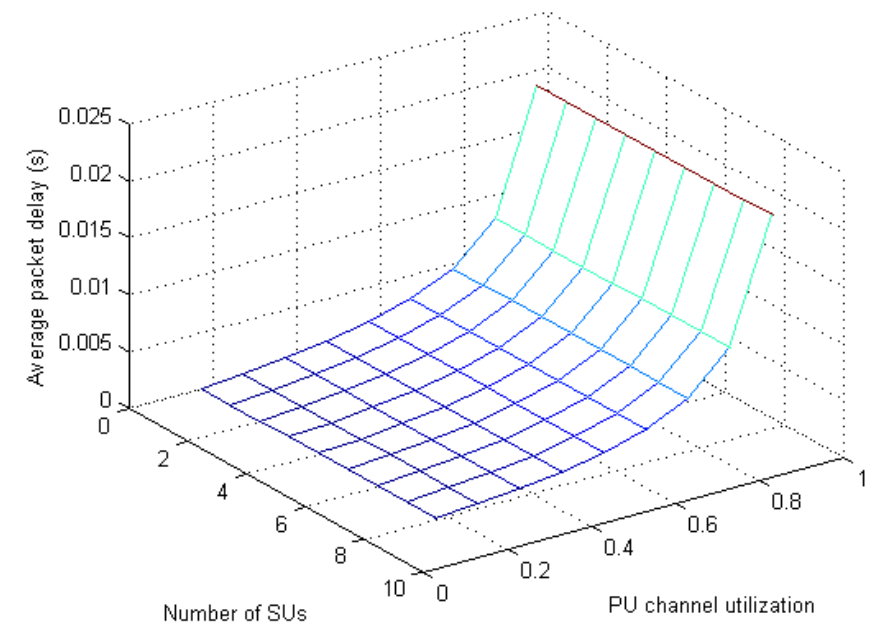

(b) $N_{p u}=10, N_{s u}=1$ to 9 by 1 , and $\lambda=5$ to 45 by 5

Fig. 6. Average packet delay versus PU channel utilization.

Last, in this section, the network lifetime is evaluated to verify the energy efficiency of ENC-MAC. We also evaluated the performance of ECRQ-MAC [19], as well as O-MAC [15] and OMC-MAC [16], because it is one of the hybrid protocols that focuses on energy efficiency. Fig. 7 shows the network lifetime versus PU channel utilization for the MAC protocols. In order to evaluate the MAC protocols under the same conditions, we set $T_{s}$ and $T_{m s}$ to $100 \mathrm{~ms}$ and $1 \mathrm{~ms}$, respectively.

As expected, it is depicted in Fig. 7 that the SUs in ENC-MAC ran out of energy later than in other MAC protocols. There are several reasons. First, the ENC-MAC sensing phase is relatively shorter than the others, and thus energy is saved. In ENC-MAC, which maintains the states of all data channels through cooperative sensing, only two minislots are required to sense two data channels, i.e., the CFR and the CFU, at the beginning of a timeslot, while at least $N_{p u}$ minislots in the timeslot are needed in O-MAC, OMC-MAC, and ECRQ-MAC to sense all data channels. Especially, the energy is wasted in O-MAC because only the assigned minislot in the reporting phase is available for an SU. 
Second, ENC-MAC has a quite longer data transmission phase in the timeslot than the other protocols. Note that the contention phase only exists on data channels in OMC-MAC and ECRQ-MAC, because only a single transceiver is available for an SU. In OMC-MAC, the length of the contention phase is varied according to the available number of data channels. On the other hand, in ENC-MAC and O-MAC, the contention is carried out over the control channel by use of another transceiver, i.e., a control transceiver. This means that ENC-MAC and O-MAC secure more resources than OMC-MAC and ECRQ-MAC. Because the data transmission phase of the aforementioned protocols is relatively shorter than that of ENC-MAC, they spend more time to sense data channels. As illustrated in Fig. 7, the network lifetime of ENC-MAC is remarkable when the number of available PU channels becomes small, i.e., high PU channel utilization. This is because the SUs that do not reserve the data channels enter the doze state.

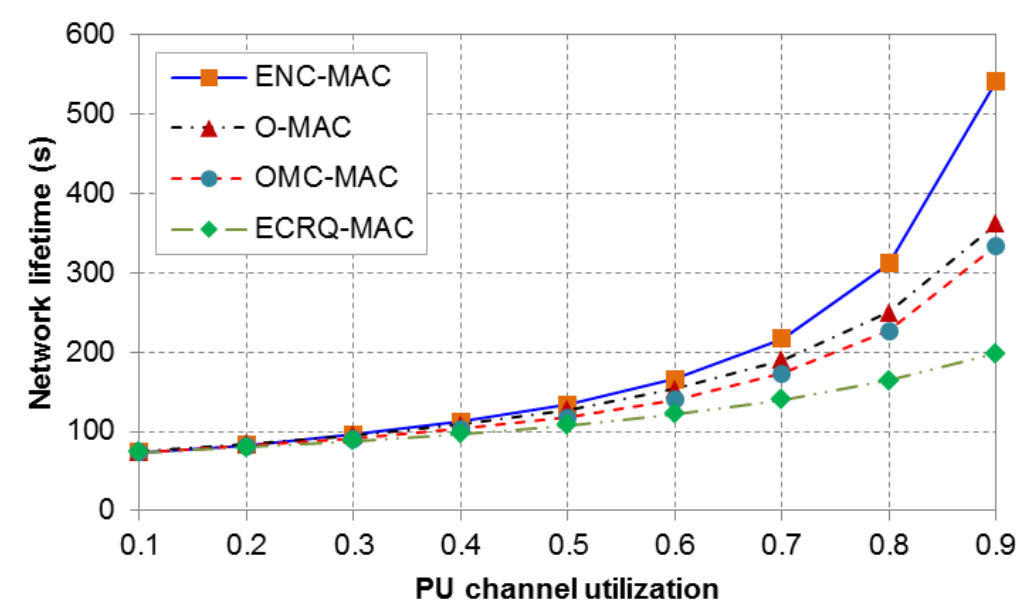

Fig. 7. Network lifetime versus PU channel utilization for ENC-MAC, O-MAC, OMC-MAC, and ECRQ-MAC, where $T_{s}=0.1$ and $T_{m s}=0.001$.

\section{Conclusion}

Since the introduction of IoT, the importance of WSN technologies has increased. In order to guarantee the resources in WSNs in which the demand for various applications increases continuously, MAC protocols are utilized. A CR technology is considered as a solution for the problems in this future wireless network. However, CR MAC protocols have not yet been optimized to be energy efficient. In this paper, we proposed an energy-efficient non-overlapping channel MAC (ENC-MAC) for the CRSNs. In order to improve the spectrum efficiency, which is the first objective of the CRNs, a hybrid MAC protocol with a dedicated control channel approach was proposed to reserve the data channels in a non-overlapping manner. Moreover, cooperative spectrum sensing that allows an SU to use only two minislots in the sensing phase was proposed to reduce the energy waste for spectrum sensing. Additionally, an analytical model for evaluating the performance of ENC-MAC was developed in terms of not only throughput for spectrum efficiency, but also network lifetime for energy efficiency. As a result, it was observed that ENC-MAC outperformed the existing CR MAC protocols. In spite of the challenges, it is essential for ENC-MAC to guarantee the delivery of 
real-time traffic, such as voice and low-rate video, and provide reliable communication with minimum latency.

\section{References}

[1] L. Atzori, A. Lera, and G. Morabito, "The Internet of Things: A survey," Computer Networks (Elsevier), vol. 54, no. 15, pp. 2787-2805, Oct. 2010. Article (CrossRef Link).

[2] O. Vermesan and P. Friess, "Internet of Things - Global Technological and Societal Trends," River Publishers, May 2011.

[3] D. Miorandi, S. Sicari, F. D. Pellegrini, and I. Chlamtac, "Internet of things: Vision, applications and research challenges," Ad Hoc Networks (Elsevier), vol. 10, no. 7, pp. 1497-1516, Sept. 2012. Article (CrossRef Link).

[4] P. Huang, S. Soltani, M. W. Mutka, and N. Xi, "The Evolution of MAC Protocols in Wireless Sensor Networks: A Survey," IEEE Commun. Surveys Tuts., vol. 15, no. 1, pp 101-120, 1Q. 2013. Article (CrossRef Link).

[5] R. V. Kulkarni, A. Forster, and G. K. Venayagamoorthy, "Computational Intelligence in Wireless Sensor Networks: A Survey,” IEEE Commun. Surveys Tuts., vol. 13, no. 1, pp. 68-96, 1Q. 2011. Article (CrossRef Link).

[6] T. Watteyne, A. Molinaro, M. G. Richichi, and M. Dohler, "From MANET To IETF ROLL Standardization: A Paradigm Shift in WSN Routing Protocols," IEEE Commun. Surveys Tuts., vol. 13, no. 4, pp. 688-707, 4Q. 2011. Article (CrossRef Link).

[7] D. Evans, "The internet of things: How the next evolution of the internet is changing everything," CISCO white paper, 2011.

[8] Z. Liang, S. Feng, D. Zhao, and X. Shen, "Delay Performance Analysis for Supporting Real-Time Traffic in a Cognitive Radio Sensor Network," IEEE Trans. Wireless Commun., vol. 10, no. 1, pp. 325-335, Jan. 2011. Article (CrossRef Link).

[9] I. F. Akyildiz, W. Y. Lee, and K. R. Chowdhury, "CRAHNs: Cognitive radio ad hoc networks," Ad Hoc Networks (Elsevier), vol. 7, no. 5, pp. 810-836, Jul. 2009. Article (CrossRef Link).

[10] P. Ren, Y. Wang, Q. Du, and J. Xu, "A survey on dynamic spectrum access protocols for distributed cognitive wireless networks," EURASIP Journal on Wireless Communications and Networking, vol. 2012, no. 60, Feb. 2012. Article (CrossRef Link).

[11] M. A. Yigitel, O. D. Incel, and C. Ersoy, "QoS-aware MAC protocols for wireless sensor networks: A survey," Computer Networks (Elsevier), vol. 55, no. 8, pp. 1982-2004, Jun. 2011. Article (CrossRef Link).

[12] S. D. T. Kelly, N. K. Suryadevara, and C. Mukhopadhyay, "Towards the Implementation of IoT for Environmental Condition Monitoring in Homes," IEEE Sensors J., vol. 13, no. 10, pp. 3846-3853, Oct. 2013. Article (CrossRef Link).

[13] C. Cormio and K. R. Chowdhury, "A survey on MAC protocols for cognitive radio networks," Ad Hoc Networks (Elsevier), vol. 7, no. 7, pp. 1315-1329, Sept. 2009. Article (CrossRef Link).

[14] N. Faruk, M. I. Gumel, A. A. Oloyode, and A. A. Ayeni, "Performance Analysis of Hybrid Protocol for Cognitive Radio Networks," Int. J. Communications, Network and System Sciences, vol. 6, no. 1, pp. 18-28, Jan. 2013. Article (CrossRef Link).

[15] H. Su and X. Zhang, "Cross-Layer Based Opportunistic MAC Protocols for QoS Provisioning Over Cognitive Radio Wireless Networks," IEEE J. Sel. Areas Commun., vol. 26, no. 1, pp. 118-129, Jan. 2008. Article (CrossRef Link).

[16] S. C. Jha, U. Phuyal, M. M. Rashid, and V. K. Bhargava, "Design of OMC-MAC: An Opportunistic Multi-Channel MAC with QoS Provisioning for Distributed Cognitive Radio Networks," IEEE Trans. Wireless Commun., vol. 10, no. 10, pp. 3414-3425, Oct. 2011. Article (CrossRef Link).

[17] G. N. Iyer and Y. C. Lim, "Efficient multi-channel MAC protocol and channel allocation schemes for TDMA based cognitive radio networks," in Proc. IEEE ICCSP '11, pp. 394-398, Feb. 2011. Article (CrossRef Link). 
[18] M. Timmers, S. Pollin, A. Dejonghe, L. Van der Perre, and F. Atthoor, "A distributed multichannel MAC protocol for multihop cognitive radio network," IEEE Trans. Veh. Tech., vol. 59, nol. 1, pp. 446-459, Jan. 2010. Article (CrossRef Link).

[19] S. M. Kamaruzzaman, and M. A. Hamid, and M. Abdullah-Al-Wadud, "An energy-efficient MAC protocol for QoS provisioning in cognitive radio ad hoc networks," Radioengineering, vol. 19, no. 4, pp. 567-578, Dec. 2010.

[20] G. P. Joshi, S. Y. Nam, and S. W. Kim, "Decentralized Predictive MAC Protocol for Ad Hoc Cognitive Radio Networks,” Wireless Pers. Commun., vol. 74, no. 2, pp. 803-821, Jan. 2014. Article (CrossRef Link).

[21] P. Cheng, R. Deng, and J. Chen, "Energy-Efficiency Cooperative Spectrum Sensing in Sensor-Aided Cognitive Radio Networks," IEEE Wireless Commun., vol. 19, no. 6, pp. 100-105, Dec. 2012. Article (CrossRef Link).

[22] S. H. Kown, B. S. Kim, and B. H. Roh, "Preemptive Opportunistic MAC Protocol in Distributed Cognitive Radio Networks,” IEEE Commun. Lett., vol. 18, no. 7, pp. 1155-1158, Jul. 2014. Article (CrossRef Link).

[23] J. Mo. H. -S. W. So, and J. Walrand, "Comparison of Multichannel MAC Protocols," IEEE Trans. Mobile Comput., vol. 7, no. 1, pp. 50-65, Jan. 2008. Article (CrossRef Link).

[24] A. D. Domenico, E. C. Strinati, and M. -G. Di Benedetto, "A Survey on MAC Strategies for Cognitive Radio Networks," IEEE Commun. Survey Tuts., vol. 14, no. 1, pp. 21-43, 1Q. 2012. Article (CrossRef Link).

[25] IEEE Standard 802.11-2012, "Wireless LAN Medium Access Control (MAC) and Physical Layer (PHY) Specifications," Mar. 2012.

[26] I. F. Akyildiz, W. Su, Y. Sankarasubramaniam, and E. Cayirci, "Wireless sensor networks: a survey," Computer Networks (Elsevier), vol. 38, no. 4, pp. 393-422, Mar. 2002. Article (CrossRef Link).

[27] B. S. Kim, G. M. Lee, and B. H. Roh, "ASPD: Adaptive Sensing Period Decision for Time-Varying Channel in Military MANETs," in Proc. IEEE MILCOM '14, pp. 643-648, Oct. 2014. Article (CrossRef Link).

[28] W. Y. Lee and I. F. Akyildiz, "Optimal spectrum sensing framework for cognitive radio networks," IEEE Trans. Wireless Commun., vol. 7, no. 10, pp. 3845-3857, Oct. 2008. Article (CrossRef Link).

[29] Y. Liu, R. Yu, and S. Xie, "Optimal Cooperative Sensing Scheme Under Time-Varying Channel for Cognitive Radio Networks,” in Proc. of IEEE DySPAN ’08, Oct. 2008. Article (CrossRef Link).

[30] IEEE Standard 802.22-2011, "Part 22: Cognitive Wireless RAN Medium Access Control (MAC) and Physical Layer (PHY) Specifications: Policies and procedures for operation in the TV Bands," 2011.

[31] S. Borst, "User-level performance of channel-aware scheduling algorithms in wireless data networks," in Proc. of IEEE INFOCOM '03, pp. 321-331, Mar.-Apr. 2003. Article (CrossRef Link).

[32] Bianchi, "Performance analysis of the IEEE 802.11 distributed coordination function," IEEE J. Sel. Areas Commun., vol. 18, no. 3, pp. 535-547, Mar. 2000. Article (CrossRef Link).

[33] H. P. Shiang, M. van der Schaar, "Queuing-Based Dynamic Channel Selection for Heterogeneous Multimedia Applications Over Cognitive Radio Networks," IEEE Trans. Multimedia, vol. 10, no. 5, pp. 896-909, Jun. 2008. Article (CrossRef Link).

[34] M. Elasaadany, M. Abdallah, T. Khattab, and M. Khairy, "Cognitive Relaying in Wireless Sensor Networks: Performance Analysis and Optimization," in Proc. of IEEE GLOBECOM '10, Dec. 2010. Article (CrossRef Link). 


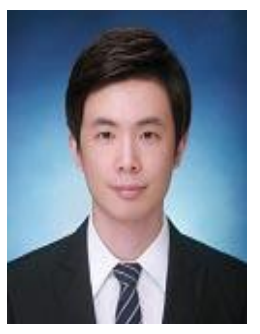

Bosung Kim received a B.S degree in Information and Computer Engineering and a Ph.D. degree in Computer Engineering from Ajou University, Suwon, Korea, in 2009 and 2015, respectively. From March 2015, he is currently a Senior Researcher in Information Communication Institute, Ajou University, Suwon, Korea. His research interests include cognitive radio (CR) communications, Internet of Things (IoT), military communications, multimedia quality of service (QoS), satellite communications, and wireless sensor networks (WSN).

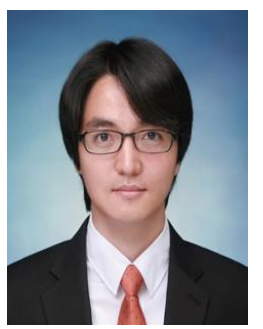

Kwangsoo Kim received a B.S. degree in Information and Computer Engineering from Ajou University, Suwon, Korea, in 2009. He is currently a Ph.D. candidate in the Department of Computer Engineering, Graduate School, Ajou University, Suwon, Korea since 2009. His research interests include mobile multimedia networking, Content Centric Network (CCN), network QoS, and tactical network.

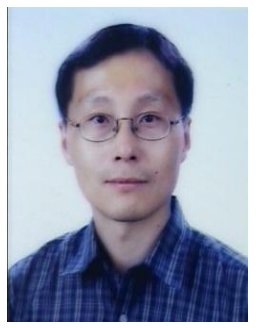

Byeong-hee Roh received a B.S. degree in Electronics Engineering from Hanyang University, Seoul, Korea, in 1987, and M.S. and Ph.D. degrees in Electrical Engineering from Korea Advanced Institute of Science and Technology (KAIST), Daejeon, Korea, in 1989 and 1998, respectively. From 1989 to 1994, he was with Telecommunication Networks Laboratory, Korea Telecom, as a researcher. From February 1998 to March 2000, he worked with Samsung Electronics Co., Ltd., Korea, as a Senior Engineer. Since March 2000, he has been with the department of Software Convergence Technology, and the department of Computer Engineering, Graduate School, Ajou University, Suwon, Korea, where he is currently a professor. During 2005, he was a visiting associate professor at Dept. of Computer Science, State University of New York, at Stony Brook, New York, USA. During 2014, he was an adjunct researcher at ADD (Agency for Defense Development), Korea. His research interests include mobile multimedia networking, future Internet network, IoT (Internet of Things) platform and services, network security and cyber warfare, and military communications. 